\title{
tic\&société
}

Vol. 14, $\mathbf{N}^{\circ}$ 1-2 | 1er semestre 2020 - 2ème semestre 2020

Mutations numériques de la musique : des

contradictions à analyser

\section{Compétences communicationnelles et pratiques numériques des « musiciens connectés »}

Communication skills and digital practices of "connected musicians"

Competencias comunicacionales y prácticas digitales de los "músicos

conectados"

Stéphane COSTANTINI

\section{CpenEdition}

Journals

Édition électronique

URL : https://journals.openedition.org/ticetsociete/4833

DOI : 10.4000/ticetsociete.4833

Éditeur

Association ARTIC

Édition imprimée

Pagination : 131-157

Référence électronique

Stéphane COSTANTINI, « Compétences communicationnelles et pratiques numériques des

" musiciens connectés » », tic\&société [En ligne], Vol. 14, № 1-2 I 1er semestre 2020 - 2ème semestre

2020, mis en ligne le 11 novembre 2020, consulté le 28 juin 2022. URL : http://

journals.openedition.org/ticetsociete/4833; DOI : https://doi.org/10.4000/ticetsociete.4833

This work is licensed under a Creative Commons Attribution-NonCommercial-NoDerivatives 4.0 International License. 
tic\&société - 14(1-2), 2020

\title{
Compétences communicationnelles et pratiques numériques des « musiciens connectés »
}

\begin{abstract}
Stéphane COSTANTINI
Stéphane Costantini est docteur en sciences de la communication, membre du Centre Georg Simmel (EHESS) et du LabSIC (Université Paris 13). II est actuellement postdoctorant au sein du projet ANR AFRINUM. Ses travaux portent sur les stratégies d'acteurs industriels et les usages dans le domaine du numérique et du web collaboratif, ainsi que sur les pratiques des amateurs et des professionnels dans les mondes de la musique.steph.costantini@gmail.com
\end{abstract}


Compétences communicationnelles et pratiques numériques des « musiciens connectés »

\title{
Compétences communicationnelles et pratiques numériques des « musiciens connectés »
}

\begin{abstract}
Résumé : Depuis les années 2000, l'émergence de nouveaux acteurs issus des industries de la communication a été suivie de profonds bouleversements au sein des industries de la musique. Cet article propose d'interroger les enjeux qu'implique chez les musiciens la formation de compétences communicationnelles sur Internet et les réseaux socionumériques. Le déploiement par les musiciens de logiques d'actions qui tendent à se rapprocher de celles de l'animation de communauté fait apparaître de nouveaux enjeux dans la diffusion et la promotion des activités musicales. Afin de cerner ces enjeux, le travail empirique mené s'est notamment appuyé sur une analyse des discours et des pratiques des musiciens en France et au Royaume-Uni. Les résultats de ces analyses suggèrent que les pratiques communicationnelles des musiciens observés les incitent à adopter une posture d'entrepreneur de leur visibilité, une posture qui se révèle controversée à plus d'un égard.
\end{abstract}

Mots-clés : musiciens, compétences communicationnelles, réseaux socionumériques, animation de communauté, logiques d'action.

\section{Communication skills and digital practices of "connected musicians"}

\begin{abstract}
Since the 2000s, the emergence of new players from the communication industries has provoked profound upheavals within the music industries. This paper examines the challenges faced by musicians in developing communication skills on the Internet and digital social networks. Their deployment of rationale for action similar to those used in community management highlights new challenges for the dissemination and promotion of musical activities. An empirical analysis of musicians' discourse and practices in France and the United Kingdom suggests that
\end{abstract}




\title{
Stéphane COSTANTINI
}

the communication practices of the musicians encourage them to adopt an entrepreneurial stance regarding their visibility. This stance is controversial in more than one respect.

Keywords: musicians, communication skills, digital social networks, community management, rationales for action.

\section{Competencias comunicacionales y prácticas digitales de los "músicos conectados"}

\begin{abstract}
Resumen: El cambio de milenio ha significado el surgimiento de nuevos actores procedentes de las industrias de comunicación, los cuales han transformado, de manera profunda, las industrias de la música. El presente texto propone una reflexión sobre los retos que plantea a los músicos la formación en competencias comunicativas en Internet y en las redes sociales. El despliegue por los músicos de lógicas relacionadas con la animación de la comunidad que les rodea, implica el surgimiento de nuevos desafíos en la difusión y en la promoción de las actividades musicales. Para identificar estos retos, se utiliza un trabajo empírico realizado a partir de un análisis de los discursos y de las prácticas de músicos de Francia y Reino Unido. Los resultados de este análisis sugieren que las prácticas comunicativas de los músicos les obliga a tomar una postura de empresario-gestor de su visibilidad. Una postura que no está exenta de controversia.
\end{abstract}

Palabras clave: músicos, competencias comunicativas, redes sociales, animación de comunidad, lógicas de acción. 
tic\&société - 14(1-2), 2020

Le présent article reprend plusieurs éléments issus de notre recherche doctorale interrogeant les logiques socioéconomiques et les pratiques communicationnelles des musiciens. Cette recherche tentait de mettre en lumière les rôles respectifs des musiciens et des acteurs d'Internet dans la recomposition du marché de la musique numérique, dans une dimension socioéconomique (pratiques, tactiques et stratégies) et socioanthropologique (postures et représentations). La problématique centrale sera ici envisagée à travers le questionnement de la figure du musicien-entrepreneur et celle de son alter ego sur les réseaux socionumériques : l'animateur de communauté, ou community manager.

La focalisation de notre recherche sur la communication, à travers les pratiques et les compétences des musiciens, ainsi que le choix des plateformes de contenus et des réseaux socionumériques comme objets privilégiés reposent sur le fait qu'ils constituent un aspect crucial dans les mouvements en cours. À la suite de Bernard Miège (1989), nous pouvons en effet considérer que la communication ne concerne pas uniquement la «mobilisation à large échelle de technologies informationnelles et communicationnelles ", mais " consiste tout autant dans le recours croissant - plus ou moins consciemment maîtrisé [...] - à des techniques d'organisation, de management, de circulation de l'information, de mise en relation et d'activation du "fonctionnement" des groupes » (p. 16). En ce sens, ces deux aspects de la communication, mobilisation des TIC d'un côté, recours à des techniques de "gestion » du social de l'autre, apparaissent comme " difficilement dissociables ". Au contraire, « ils s'appuient l'un sur l'autre et se complètent » (Miège, 1989, p. 17). À cet égard, il semble que le développement des réseaux socionumériques, dispositifs ${ }^{1}$ numériques qui tendent à adopter une place «centrale» dans l'économie des pratiques communicationnelles étudiées, peut être précisément envisagé sous ces deux aspects.

\footnotetext{
${ }^{1}$ Nous entendons ici dispositif au sens de Giorgio Agamben (2007), qui, élargissant la notion forgée par Foucault, désigne dispositif comme « tout ce qui a, d'une manière ou d'une autre, la capacité de capturer, d'orienter, de déterminer, d'intercepter, de modeler, de contrôler et d'assurer les gestes, les conduites, les opinions et les discours des êtres vivants » (p. 30-31).
} 


\section{Stéphane COSTANTINI}

Deux points principaux peuvent caractériser le contexte de cette étude. En premier lieu, il paraît indispensable de tenir compte des mutations des industries de la musique, traversées depuis plus de quinze ans par des influences en provenance de multinationales qui sont parfois désignées comme les "nouvelles majors » (ou GAFAM : Google, Apple, Facebook, Amazon, Microsoft) (Bouquillion, Miège et Mœglin, 2013). Avec la forte baisse des ventes de musique sur support " physique " et le développement du marché de la musique "numérique ", les dispositifs portés par ces acteurs (ainsi qu'une myriade d'autres) incitent en amont, dans les instances de la production, à de nouvelles modalités de diffusion de la musique ${ }^{2}$. De plus, les stratégies de ces acteurs se positionnent également en aval de ces pôles, en incitant les usagers et les professionnels à l'adoption d'usages qui correspondent à un renouvellement des modalités de circulation des produits culturels et informationnels ${ }^{3}$. Sous cet angle, nombre de ces acteurs se sont insérés dans des segments du marché auparavant inexploités, ou laissés en partie vacants par les acteurs historiques de la musique et des médias (Belleflamme, 2003 ; Benghozi, 2011; Benghozi et Paris, 2001 ; Beuscart, 2007).

Concernant les musiciens, ces mutations s'accompagnent d'une baisse des barrières à l'entrée du marché de la musique (Benghozi et Paris, 2001), contribuant à un brouillage des frontières entre musiciens dits " amateurs » et musiciens dits « professionnels » (Costantini, 2012 ; Coulangeon, 2004, 2008). Ceci est à mettre en lien avec la tendance, depuis les années 1980, au développement soutenu des pratiques musicales et à l'augmentation générale des effectifs des musiciens dans les pays de la Triade. Ce phénomène accompagne ainsi la multiplication des pôles de la création (home studios, microlabels) et l'avènement de l'« artiste producteur » (Jouvenet, 2006). Autrement dit, il n'y a jamais eu autant de musiciens au sein du "vivier artistique »- pour reprendre la notion de Huet et al. (1978) -, ce qui contribue au phénomène de surproduction des contenus (Menger, 2009),

\footnotetext{
${ }^{2}$ À travers l'utilisation de plateformes numériques telles que YouTube, iTunes Store et Apple Music, mais également de services tels que Spotify, Deezer et Soundcloud.

3 Notamment à travers des listes de lectures numériques, des blogues, des webzines et, naturellement, des réseaux socionumériques tels Facebook ou Twitter, mais aussi YouTube ou Soundcloud, qui incluent certaines fonctionnalités similaires (commentaires, reposts).
} 
Compétences communicationnelles et pratiques numériques des « musiciens connectés »

rend particulièrement stratégiques sa gestion et sa curation, mais pose aussi inévitablement la question de la précarisation de ce vivier.

En second lieu, avec l'adoption massive des plateformes de contenus numériques et des réseaux socionumériques, on assiste à l'extension progressive des signes de reconnaissance artistique aux métriques et aux compteurs relationnels proposés par les dispositifs numériques (nombre d'écoutes ou de visionnages de vidéos, nombre d'amis, de fans, d'abonnés) et l'émergence d'une notoriété en ligne (Beuscart, 2008). Phénomène notable, les professionnels du marketing au sein de la filière de la musique enregistrée mobilisent quotidiennement les dispositifs numériques développés par ces nouveaux acteurs, et ce, tant en amont (recherche de nouveaux talents ou genres musicaux) qu'en aval (diffusion et promotion de contenus). Mais surtout, ces professionnels tentent de façon croissante d'articuler ces deux pôles en maximisant les relations entre marketing amont et aval. Si l'emprise du marketing sur cette filière est davantage une tendance de fond qu'un phénomène récent (Negus, 1999), on peut tout de même constater que le développement du marché de la musique numérique tend à amplifier les logiques rationalisantes au sein de la filière (Hesmondhalgh, 2007). Ce développement est également l'occasion d'une réorganisation des corps de métiers des labels, avec l'apparition de fonctions de gestionnaires de la relation client (CRM), de webmarketeurs ou de community managers. L'adoption progressive des dispositifs de distribution et de promotion en ligne, par un ensemble grandissant d'acteurs des industries de la musique, a en ce sens activement concouru à la montée en puissance des phénomènes symétriques d'injonction à la visibilité et d'impératif de communication.

À l'égard de ces constats, nos recherches doctorales (Costantini, 2014) ont pu suggérer que les musiciens autoproduits ou signés au sein de petits labels utilisent en partie les mêmes moyens de diffusion et de promotion en ligne et sont donc pareillement soumis à un impératif de communication, porteur de nombreuses injonctions. Dans ce cadre, les musiciens sont par extension placés en position de garants de leur visibilité médiatisée, « qui engage l'intervention de tiers sous forme de supports symboliques, de techniques, d'images ou de sons " (Voirol, 2005, p. 98). Ces injonctions se retrouvent par 


\section{Stéphane COSTANTINI}

ailleurs de façon très marquée et visible dans le cas des plateformes de financement participatif, où les musiciens " endossent les rôles additionnels de producteurs ou d'éditeurs et, surtout, de marketeurs, de promoteurs ou de communicants ॥ (Dupuy-Salle et Schmitt, 2019, p. 117). Aussi peut-on voir chez ceux-ci la formation et le déploiement de compétences communicationnelles, entendues comme un ensemble de "procédures " et de "savoir-faire susceptibles de faire écho aux schèmes d'intelligibilité " (Voirol, 2005, p. 110) de leurs destinataires potentiels (publics et professionnels). Ces compétences ne sont pas, pour la plupart, directement reliées à l'émergence des TIC, mais elles sont plutôt redéployées à l'occasion de leur appropriation. L'acquisition et la mobilisation de ces compétences permettent notamment aux musiciens d'élargir considérablement leurs registres d'actions disponibles, ce qui favorise la gestion autonome et le développement de leurs activités musicales. Ce mouvement peut également leur permettre d'augmenter ou de maintenir leurs chances d'employabilité au sein du secteur musical, ou bien dans d'autres secteurs où l'on valorise ces compétences. En ce sens, l'augmentation des effectifs de musiciens, et la concurrence produite par ce brouillage des frontières entre amateurs et professionnels (parfois à l'avantage des premiers), s'accompagne de nouveaux enjeux entourant les compétences et les carrières. Comme le remarque Pierre-Jean Benghozi (2011) à ce propos, " [l]es futurs professionnels s'interrogent sur l'intérêt, mais aussi la possibilité même d'investir individuellement temps et énergie dans le développement de capacités, d'expériences et de pratiques qui ne seront ultérieurement que difficilement valorisables » (p. 9). Aussi le questionnement central qui traverse cet article tente de déterminer dans quelle mesure les pratiques numériques et les discours des musiciens reflètent les changements en cours, notamment dans la redistribution des cartes entre acteurs historiques des contenus et "nouveaux" acteurs de l'intermédiation numérique. Après un bref rappel du vocabulaire spécifique utilisé, nous envisagerons ce questionnement à travers trois logiques d'action spécifiques qui caractérisent la posture du musicien en tant que community manager: la «facilitation d'accessibilité », l'« esthétisation de l'impératif communicationnel » et la «promotion socialisante ». 
Compétences communicationnelles et pratiques numériques des « musiciens connectés »

La méthodologie employée durant cette recherche a consisté à réaliser, entre 2010 et 2012, une série de longs entretiens semi-directifs auprès de 35 musiciens, dont les trajectoires sont très variées ${ }^{4}$, tout comme leur inscription territoriale (moyennes et grandes villes de France et du Royaume-Uni). Néanmoins, ils ont tous pour point commun l'utilisation d'Internet en tant qu'outil de diffusion, de distribution et de promotion d'une part et, d'autre part, les genres pratiqués, soit les musiques rock et électronique. Cette étude contrastive, qui prend de façon assumée les musiciens comme point d'entrée, a pour objectif de repérer les différents registres d'action mis en œuvre dans le cadre de leurs pratiques numériques pour le développement et la continuation de leur pratique musicale. Les discours et les témoignages produits dans ce cadre ont été croisés avec un suivi des pratiques communicationnelles de ces musiciens sur Internet et les réseaux socionumériques. La période de ces entretiens, il faut le reconnaître, peut apparaître sensiblement datée, tant elle fût dans le même temps marquée par un contexte de changements intenses des usages du numérique dans les deux territoires observés (notamment marquée par l'achèvement de la " migration » des musiciens de la plateforme MySpace vers celle de Facebook, par l'intensification de l'usage du smartphone et par la mise en place progressive d'une régie publicitaire sur Facebook). Pour autant, le suivi (bien que de façon moins constante) des pratiques de ces musiciens sur les réseaux de diffusion, de vente et de promotion musicale n'ont pas invalidé les hypothèses et les conclusions formulées ici. Au contraire, les tendances observées semblent s'être renforcées au cours de la période ultérieure au temps de notre analyse - entre autres avec l'adoption de réseaux socionumériques tels qu'Instagram ou Snapchat. Nous reviendrons succinctement sur ces points au cours et en conclusion de cet article.

\footnotetext{
${ }^{4}$ Les musiciens étudiés se trouvent en grande partie autoproduits ou signés au sein de petits labels, leur projet musicaux pouvant être désignés « en développement " par les professionnels du secteur (Guibert et Sagot-Duvauroux, 2013). En ce qui concerne l'entourage professionnel, certains sont signés dans une agence de diffusion (booking) ou un éditeur musical (publishing), quand d'autres ont monté leurs propres structures ou assurent eux-mêmes ces fonctions. Ces situations recouvrent toutefois une pluralité de trajectoires socioprofessionnelles: certains sont des musiciens monoactifs (intermittents du spectacle ou auto-entrepreneurs "à plein temps »), d'autres sont pluriactifs et exercent une activité au sein du secteur musical ou à l'extérieur de celuici, d'autres encore sont étudiants ou au chômage.
} 


\section{Stéphane COSTANTINI}

\section{Des logiques d'action et des univers de reconnaissance différenciés}

Pour tenir compte des pratiques communicationnelles sur les dispositifs numériques du Web collaboratif des musiciens, il semble pertinent d'envisager le déploiement de ces compétences sous forme de logiques d'action. Cette notion permet de saisir les pratiques des musiciens dans un mouvement dialectique réflexif, entre finalités rationnelles - la recherche du succès - et coopérations, qui correspondent à l' " attitude inter-compréhensive " formalisée par Habermas (1987). Cette notion permet, dans ce cadre, « de rendre compte des différents niveaux où s'enracinent les comportements des acteurs - seraient-ils contradictoires » (Bernoux et al., 2005, p. 203). En effet, l'analyse des pratiques et des discours, comme nous le verrons infra, nous a imposé de constater que les musiciens ne s'autoqualifiaient (à juste titre) que très rarement de "stratèges " du marketing numérique, ni de "victimes " de celui-ci, tendant à intégrer et à négocier au mieux avec les usages possibles des plateformes et ceux des internautes afin de parvenir, parfois, à "tirer [leur] épingle du jeu » comme l'a verbalisé l'un d'entre eux.

La qualification de ces logiques d'action nous amène à faire la distinction entre deux ensembles de pratiques de diffusion et de promotion des activités des musiciens sur les réseaux socionumériques.

Les pratiques de diffusion et de promotion d'un premier ensemble de pratiques peuvent être qualifiées de " normatives ", car calquées sur le modèle mass-médiatique en vigueur dans la promotion musicale. II consiste principalement, pour les musiciens, en la publication de leurs productions musicales et d'annonces promotionnelles à propos des activités du projet musical : dates de concerts, sorties de disques ou de nouveaux titres, articles de presse, endorsement par une marque, synchronisation publicitaire, etc. Ce type de pratiques est le plus couramment rencontré sur Facebook, une grande partie des musiciens se contentant d'un usage relativement restreint de publications et de création d'évènements. La régularité des publications dépend alors en grande partie de l'intensité des activités musicales des protagonistes.

Les pratiques du second ensemble peuvent être qualifiées, 
Compétences communicationnelles et pratiques numériques des « musiciens connectés »

pour leur part, de «socialisantes", car composées de publications et d'interactions médiatisées plus ciblées, visant à susciter l'intérêt des internautes, à les faire réagir à leurs publications et, in fine, à augmenter, sur la plateforme, la circulation des contenus et des informations les concernant. Contrairement au premier type de pratiques, celles-ci se trouvent davantage déconnectées des activités de production et de diffusion des musiciens. Elles se caractérisent par la recherche de la création ou du maintien d'une relation entre artistes et auditeurs par l'intermédiaire du dispositif, démarche que l'on peut assimiler à une tentative de fidélisation de l'auditoire.

Ces deux ensembles de pratiques se déclinent sous la forme de trois logiques d'action qui vont caractériser le déploiement des compétences communicationnelles des musiciens. Toutefois, il faut préciser que ces pratiques en ligne ne correspondent jamais exactement à l'un ou l'autre des ensembles, présentés ici sous une forme "idéal-typique », mais que chacune d'entre elles emprunte plus ou moins à ces deux formes de communication, parfois de façon synchrone.

Une seconde volonté de clarification terminologique traverse cette recherche : il nous semble nécessaire, dans le contexte de l'étude des réseaux socionumériques, de déconstruire la notion de visibilité qui, englobante, ne donne aucune indication sur les agents producteurs de cette visibilité (le «public» des internautes) ni sur leur " qualité » ou même sur la nature de leur intérêt. Les travaux de Jean-Samuel Beuscart offrent à cet égard un point de départ heuristique en considérant la notion de notoriété comme un capital, «à l'obsolescence forte et susceptible d'être valorisée sur plusieurs marchés » (Beuscart, 2008), et en la dissociant en deux composantes distinctes: " l'audience, qui est une somme d'actes de consommations et témoigne du fait d'être écouté ; et l'influence, qui témoigne du fait d'être recommandé activement par son public, et traduit un attachement du public à l'artiste allant au-delà de la simple écoute » (Beuscart et Couronné, 2009, p. 148). Dans son observation du réseau socionumérique MySpace, il remarque en outre que son " lieu de construction et d'objectivation » (p. 148) se trouve désormais autant en ligne que dans les classements de ventes (physique ou numérique) ou les taux de remplissage des salles de concert.

À la suite de ces travaux et dans le cadre de l'analyse des 


\section{Stéphane COSTANTINI}

pratiques socioéconomiques et médiatiques des musiciens, nous proposons de distinguer quatre " univers de reconnaissance " : celui du monde de la musique (la réputation), celui des spécialistes du domaine (la notoriété), celui du grand public (la popularité) et celui des instances officielles de consécration artistique (le prestige) ${ }^{5}$. Les traces et les autres artéfacts produits par les plateformes de networking social, par l'intermédiaire des usages des internautes, tendent à amalgamer de facto ces quatre univers sous le sceau de la "visibilité »... suggérant de façon implicite que ces différents univers sont tout à fait interchangeables puisqu'ils participent à la valorisation de l'image des artistes par l'intermédiaire de ses diverses productions et publications et les inscrivant plus aisément dans une économie de l'attention qui structure fortement les modèles économiques des GAFA (Citton, 2012, 2013).

C'est précisément cette simplification de la réalité sociale des pratiques qu'il nous semble important de déconstruire, notamment parce qu'elle reflète des enjeux idéologiques pour les plateformes observées, mais également qu'elle détourne d'une appréhension fine des pratiques des musiciens. Aussi, il convient à notre sens d'examiner plus précisément quelles interférences existent de l'un à l'autre de ces univers de reconnaissance dans les stratégies de production des valeurs et d'homologation artistique. Comme nous le verrons plus loin, ces quatre univers peuvent être tout à fait distincts, voire opposés, à la fois dans les discours et les intentionnalités communicationnelles des musiciens étudiés.

\section{Musique en streaming et logique d'action de « facilitation d'accessibilité »}

Tout d'abord, on peut identifier chez les musiciens observés une logique d'action de «facilitation d'accessibilité », qui concerne avant tout les pratiques de diffusion de titres musicaux. Cette logique d'action consiste, pour les musiciens, à tenir compte des usages développés sur Internet et de s'en accommoder au mieux afin de tirer un avantage quant à leur "visibilité ». Comme le rappellent Gérôme Guibert et Dominique Sagot-Duvauroux (2013) au sujet de la réaction des musiciens

${ }^{5}$ Je tiens ici à remercier Pierre Mœglin pour cette suggestion de catégorisation. 
Compétences communicationnelles et pratiques numériques des « musiciens connectés »

vis-à-vis du piratage :

Les musiciens sont avant tout pragmatiques dans l'articulation disque et spectacle vivant et ils dissocient circulation des enregistrements, succès d'un disque et problématiques de tournées. De ce point de vue, le piratage des enregistrements n'est donc pas perçu comme quelque chose de négatif. [...] Le niveau de notoriété, l'activité scénique et l'autoproduction jouent donc un rôle dans la manière dont les artistes considèrent leur environnement, et l'antagonisme ou la particularité de la scène. (p. 52.)

Avec la montée en importance des plateformes de streaming (chez lesquelles la mise en ligne de titres musicaux est réservée aux producteurs identifiés) telles que Deezer et Spotify, mais également des plateformes User Generated Content (UGC) telles que YouTube ou Soundcloud, ces pratiques se sont peu ou prou généralisées dans les années 2010. En témoignent par exemple les conseils de la manageur et spécialiste du marketing musical Virginie Berger dans un manuel pratique destiné aux " musiciens marketeurs", où elle leur recommande de " disperser" gratuitement des titres en streaming et en téléchargement afin de «maximiser la découverte » (dans Berger, 2011).

Toutefois, on ne peut pas restreindre ces pratiques à des formes de coopération entre musiciens et internautes. En effet, la prise en compte des usages des internautes par les musiciens double les motivations "rationnelles " qu'ont ces derniers par rapport à leur quête de visibilité sur Internet d'une nécessité de « maîtriser » d'une part les modalités de cette visibilité et, d'autre part, l'accès aux contenus musicaux.

Il s'agit, par exemple, pour les musiciens - lorsqu'ils sont autoproduits - ou leurs labels de placer leurs musiques sur les sites de streaming pour éviter que des internautes ne le fassent un usage courant sur des sites tels que YouTube ou SoundCloud - ou aient recours au téléchargement illégal. Cela peut aussi consister à ne diffuser que des extraits de morceaux ou d'albums en vue de réduire leur valeur d'usage et d'encourager leur téléchargement légal. Ces pratiques sont assez symptomatiques de l'autocontrôle opéré par certains musiciens sur Internet, qui ont connaissance des usages développés par leurs propres auditeurs. Elles montrent en 


\section{Stéphane COSTANTINI}

quelque sorte la proximité et les formes de solidarité entre musiciens-producteurs et labels, mais aussi l'adaptation aux mondes du numérique des formes de rationalisation propres à la filière de la musique enregistrée, en l'occurrence, ici, la gestion de l'accès aux contenus musicaux. Sous cet angle, cette logique d'action de facilitation d'accessibilité apparaît plutôt comme normative et soumise à la fois aux injonctions des acteurs du Web (diffusion élargie des contenus) et de ceux de la musique enregistrée (gestion et contrôle de leur accès). À cette aune, on peut remarquer que la logique de facilitation d'accessibilité, qui semble s'être considérablement développée dans le cadre des interconnexions croissantes entre plateformes de contenus et sites de réseautage social, entretient de fortes connivences idéologiques avec le paradigme industriel de la convergence, tel que l'a identifié Philippe Bouquillion. Au sein de ce paradigme, en effet, " le rapport des agents sociaux à la culture est placé sous le signe de l'accès », notamment à travers la " possibilité pour les consommateurs d'échapper aux logiques de programmation [des médias traditionnels] pour choisir euxmêmes dans un panel de contenus ce qu'ils désirent effectivement consommer " (Bouquillion, Miège et Mœglin, 2013, p. 42). Or, et comme le remarque Vincent Bullich (2015), ce paradigme concourt dans le même temps

au remplacement, ou tout du moins, à la concurrence des mythes de « l'auteur romantique » et de « l'œuvre reflet de la personnalité », qui constituent le socle idéel de la propriété artistique, institution cardinale dans les économies des industries culturelles [...], par les mythes de "l'auteur-multitude » ou " l'auteur en collaboration » et de « l'œuvre en partage » que portent les thuriféraires de la " culture participative » et qui constituent un des socles idéels sur lesquels s'appuient les industries médiatisantes dans leurs stratégies de « réquisition » $(p$ 82).

Porteuse de cette contradiction, l'instauration de la logique d'action de facilitation d'accessibilité, tant dans les conceptions des musiciens que celles des différents acteurs de la production musicale, tendrait dans ce cas à naturaliser ces nouvelles formes de valorisation des produits culturels, (désormais) considérées à même de tenir compte et, plus globalement, de "réguler » les usages de la musique enregistrée sur Internet. Cette logique d'action s'appuie alors avant tout sur la capacité (réelle ou non) 
Compétences communicationnelles et pratiques numériques des « musiciens connectés »

des plateformes de streaming à concourir à leur popularité (visant l'accessibilité des publics aux œuvres), notamment afin de constituer une base de fans « revendicable » par la suite, visà-vis des programmateurs des salles de concert et des festivals (permettant donc d'accroître leur notoriété). Ce faisant, elle encourage plus ou moins passivement à la mainmise des GAFAM sur les industries du contenu, la considérant somme toute comme porteuse d'externalités positives sur d'autres secteurs musicaux, moins affectés par les industries de la communication (telles que le live, l'édition musicale ou le merchandising).

En parallèle, le couplage entre sites de streaming et réseaux socionumériques fait également ressortir une nouvelle tendance, celle de privilégier la sortie de singles au détriment de la sortie d'albums $^{6}$. Dans la filière de la musique enregistrée, cette tendance semble alors reliée à l'intensification de la production audiovisuelle à destination d'Internet et répond à la nécessité d'« alimenter » les réseaux socionumériques en nouveaux contenus de façon régulière, cela notamment afin de suivre la temporalité et les modalités de visionnage qui lui sont propres.

\section{Vidéos musicales et logique d'action d'esthétisation de la contrainte communicationnelle}

L'aspect normatif des pratiques promotionnelles doit être relativisé en tenant compte d'autres types de pratiques, notamment l'utilisation croissante des vidéos musicales en streaming ces dernières années. Cette tendance, déjà largement amorcée avec l'avènement de la plateforme YouTube, semble pour partie avoir été exacerbée par la non-intégration de fonctions de streaming musical sur Facebook et la priorité donnée aux contenus visuels (photos et vidéos) sur cette dernière plateforme. Par ailleurs, et dans la période récente, l'apparition et le développement fulgurant de réseaux socionumériques spécifiquement centrés sur des contenus visuels (photos et vidéos) et les appareils mobiles, tels qu'Instagram (en phase de développement au moment de notre recherche, 1,1 milliard d'utilisateurs actifs en 2019, racheté par

${ }^{6}$ Tendance notamment repérée par Tschmuck (2017). 


\section{Stéphane COSTANTINI}

Facebook en 2012) ou Snapchat (210 millions d'utilisateurs actifs $)^{7}$, et leur rapide intégration à la panoplie des outils numériques des musiciens ont également renforcé cette tendance. Pour une majorité d'interrogés, la motivation principale d'assortir leurs morceaux de musique d'un support vidéo (clips, captation de concert ou d'émissions de radio/télévision), parfois de façon systématique, est principalement le fait de son caractère partageable, potentiellement "viral » et donc permettant d'augmenter leur popularité.

De ce point de vue, la diffusion d'un clip vidéo est envisagée tout d'abord comme un argument de " sérieux " permettant de faire preuve de la "démarche " des musiciens en direction des collaborateurs potentiels du groupe. Cet argument correspond en effet à la systématisation, sur les sites Internet et les pages Facebook des médias spécialisés, des salles de concert et des festivals, à intégrer des vidéos. Les vidéos musicales deviennent alors un atout pour se faire programmer, car elles intègrent par la suite l'ensemble des "supports de communication " utilisés par les organisations pour promouvoir leurs activités et leurs évènements et contribuent en ce sens à la notoriété des artistes. Considérons à présent cet extrait d'entretien :

C'est vrai que le clip, il nous a beaucoup servi en termes de communication, parce qu'un clip ça plaît souvent pas mal aux gens, ça donne tout de suite une idée de l'univers visuel, parce que maintenant la musique ne suffit plus vraiment à convaincre les gens, donc il y a toujours besoin d'une petite valeur ajoutée, et puis peut-être aussi parce que le fait que ce soit moi qui aie réalisé le clip, et puis vraisemblablement qu'il plaise bien aux gens, ça a été vraiment une vraie carte d'entrée. (Perrine, Peau, pluriactive, électro-pop, Grenoble)

On peut voir, ici, l'intersubjectivité qui émane de cette logique d'action, les musiciens l'orientant à la fois vers les publics et les professionnels du secteur. L'argument de « visibilité » se fonde principalement sur les usages des internautes, et à plus forte raison sur le dispositif Facebook, qu'ils soient supposés par les musiciens ou calqués sur leurs propres pratiques de

7 Sources: Instagram (s. d.). À propos de nous. Repéré à https://about.instagram. com/about-us; Statista (2020). Nomber of daily active Snapchat users from $1^{\text {st }}$ quarter 2014 to $1^{\text {st }}$ quarter 2020. Repéré à https://www.statista.com/statistics/545967/snap chat-app-daul. 
Compétences communicationnelles et pratiques numériques des « musiciens connectés »

consommation de musique. Par ailleurs, il accompagne la propension de plus en plus forte du réseau socionumérique à privilégier l'affichage de contenus visuels au sein de sa fil d'actualité, principal lieu des interactions pour les utilisateurs. À cet égard, on remarque qu'ils sont considérés à la fois négativement, certains musiciens invoquant les pratiques de « zapping ", l'attention fragile des auditeurs sur Internet « si on ne leur met pas un visuel devant le nez », et positivement, à travers la désignation de la " tendance » à regarder des vidéos et sa propension à être un outil de "communication virale », une " valeur ajoutée », voire une " carte d'entrée » dans le cas où celle-ci est appréciée.

Toutefois, l'argument de mise en visibilité ne se limite pas à une dimension "promotionnelle », mais entre plus largement dans le cadre de la production d'un univers complet et cohérent, doté d'une identité artistique propre. Dans ce sens, ce sont les qualités intrinsèques du clip vidéo en tant que forme artistique qui sont mises en valeur pour leurs capacités à générer de la sociabilité et, donc, du partage de sens.

Le cas du Record Club mis en place par le groupe B R OAD WAY permet de cerner cet aspect de façon plus précise. Lancé par les musiciens du label et collectif 6am, le Record Club consiste en la production filmée de reprises d'artistes faisant partie de leurs influences esthétiques. L'objectif affiché par le groupe est d'assurer une diffusion régulière sur leur site Internet et leur page Facebook (de faire " monter l'attente ») avant la sortie de leur album et d'un premier clip promotionnel :

Anthony - le B R OAD WAY club, [...] c'est à la fois de la communication parce que du coup, ça fait parler de B R OAD WAY. Tous les 15 jours il y a une nouvelle vidéo qui sort, mais en même temps, c'est pour proposer de l'artistique tu vois ? Donc c'est là où je trouve que, enfin, après c'est comme tout, tu peux faire une bonne ou une mauvaise utilisation de Facebook. Là je trouve que c'est la meilleure utilisation possible, c'est-à-dire que tu ne bombardes pas sans cesse d'infos dont tout le monde se fout.

J.-C. - Voilà, c'est pas mettre de la news pour mettre de la news, quand on poste un truc, c'est qu'il y a une actu, vraiment pour tenir au courant. On se met pas la pression 


\section{Stéphane COSTANTINI}

à se dire "il faut mettre un truc tous les deux jours, pour pas qu'on nous oublie ", tu vois? (Anthony et J.-C., B R OAD WAY, Pluriactifs, électro-pop, Saint-Étienne)

La mise en place de ce Record Club fait état de plusieurs volontés adressées de façon synchrone. Elle correspond à une recherche d'inscription de leur plan de communication dans les spécificités des dispositifs du Web 2.0: sur le plan de la temporalité médiatique, car il est nécessaire d' " alimenter » leur page en contenus ou en informations afin de «faire monter l'attente du public », de faire " grandir la base de fans »; mais également sur le plan de la « connectivité », là où l'exposition de leurs influences esthétiques leur a permis, notamment, dans un cas, de contacter l'un des artistes repris qui, enthousiasmé, a aussitôt "partagé » la reprise vidéo sur sa propre page Facebook. De façon tout aussi centrale, la mise en place du Record Club apparaît comme une tentative de réconciliation entre deux aspects: 1) l'impératif de communiquer, perçu comme dégradant, voire angoissant, car soumis à la crainte d'ennuyer ou d'agacer leurs auditeurs et donc d'apparaître comme inopportun ; et 2) le plaisir du travail créateur, perçu au contraire comme partageable, valorisable. Cette logique d'action est donc à envisager comme une forme d'intégration de l'artistique à la communication, ou, autrement dit, d'esthétisation de l'impératif de communication.

\section{Réseaux socionumériques et logique d'action de promotion socialisante}

La logique d'action de promotion socialisante se rapproche également d'une recherche de compromis liée à l'injonction à la visibilité dont les musiciens font l'objet. On peut à ce sujet rappeler que ces pratiques de promotion sur le Web 2.0 reposent de façon croissante sur les phénomènes d' " audienciation » (la coproduction de l'audience par elle-même), car elle nécessite «la reconnaissance d'un contenu à travers une gradation d'activités: voter, commenter, citer, relinker, embedder ou encore remixer" (Allard, 2009, p. 35). Les pratiques qui en découlent se trouvent par ailleurs fortement promues par les professionnels du marketing, conseillant notamment aux musiciens « d'encourager l'interaction avec les fans " à travers une série de techniques d'animation (poser des questions, faire 
Compétences communicationnelles et pratiques numériques des « musiciens connectés »

des sondages) ou de gratifications symboliques (remercier les supporters les plus actifs) (Berger, 2011). La plateforme Facebook procède d'injonctions très similaires à travers l'envoi régulier de messages de notification du type : "Les fans de [nom de la Page] n'ont pas reçu de nouvelles depuis longtemps. Écrivez une publication maintenant. ». Malgré ces incitations, les musiciens autoproduits, non entourés de professionnels du marketing, ne disposent que de peu de « leviers " promotionnels pour inciter leurs auditeurs à contribuer à ces phénomènes d'audienciation. Ils sont fortement tributaires des usages propres à ces auditeurs, qui peuvent s'identifier en tant que " communauté de fans ", ou non, et dont les réactions en ligne peuvent être fortement imprévisibles.

Une illustration de cette logique d'action de promotion socialisante peut être donnée avec le groupe Anavæ (pluriactifs, pop-rock, Londres). Celui-ci a pris pour habitude de récupérer et de publier sur sa page Facebook de nombreuses photos de ses fans portant les $t$-shirts à l'effigie du groupe - en prenant généralement soin de "taguer » ses fans (de mentionner en créant une URL de leur page), afin que ces photos apparaissent sur leur profil. Cette "stratégie » relève alors autant d'une activité de publication que d'une activité de "socialisation » en ligne, qui valorise la relation entre musiciens et auditeurs. Tout en poursuivant la valorisation d'un univers artistique cohérent, les membres d'Anavæ créent, par des productions de leurs fans (les photos envoyées), un contexte de sociabilité à partir de leur identité visuelle, contribuant au passage à produire un sentiment de "communauté " (ils nomment par ailleurs leurs abonnés " ærmy ", par analogie avec le nom du groupe). Dans ces conditions, on pourrait alors affirmer que ces musiciens ont su saisir les occasions offertes par les « mécanismes d'admiration mutuelle » entre utilisateurs, dont les réseaux socionumériques célèbrent la possibilité à travers leur design d'interface (Schklovski et Boyd, 2006). Dans le même temps, on voit combien, au sein des réseaux socionumériques, l'internaute peut ainsi être envisagé comme un « complice dans le marketing viral » des musiciens (Bouquillion et Matthews, 2010, p. 129). Comme le rappelle Jacob Matthews (2015), « la consommation culturelle a toujours eu une dimension collective et impliqué une certaine activité productrice de culture, de la part des récepteurs supposés "finaux" » (p.65). Reprenant les travaux de Brice Nixon suggérant que « le travail du public fait partie intégrante 


\section{Stéphane COSTANTINI}

de ce "travail concret" générant des valeurs d'usage qui permettent au capital de se reproduire » (p. 68), il note l'actualité de cette suggestion en prenant le cas de Google. L'entreprise (à l'instar de Facebook) crée en effet constamment « de nouveaux outils permettant d'exploiter le travail du public, non pas parce qu'elle y est acculée, mais parce qu'il s'agit là de "moyens relativement peu onéreux de croître en tant que capitaliste communicationnel ॥ (Nixon, 2013, cité dans Matthews, 2015, p. 68). On peut alors voir dans la logique de promotion socialisante une injonction à rendre le public " productif », où le musicien, amené à devenir gestionnaire de son image, doit entretenir une "communauté de fans » produisant de fait des traces pouvant être monétisées par les régies publicitaires des acteurs du numérique.

Par ailleurs, ces pratiques de promotion font émerger de façon problématique la question de leur pertinence, notamment à l'égard du caractère plus ou moins " privé » des informations qu'elles dévoilent. Ainsi, Pacovolume, qui affirme d'un côté être satisfait des interactions directes qu'il entretient avec ses auditeurs, paraît, de l'autre, clairement opposé à la divulgation de détails plus ou moins personnels sur Internet, tels que le pratiquent des artistes célèbres qu'il apprécie :

Quand j'écoutais des albums quand j'étais ado, j'avais aucune information sur la vie des mecs, enfin je regardais la pochette des disques, quand c'était des 33t je me disais "tiens ça a été enregistré là ", et y avait du mystère tu vois. Et maintenant c'est le même mec, t'as un site Internet, et tu peux voir qu'il mange des Kellogg le matin, tu peux vraiment avoir des détails sur leur vie ; mais moi je m'en fous. [...] tout savoir, ou tout livrer, je trouve que ça gâche un peu la magie de la musique, c'est-à-dire ce côté où " tu ne sais pas ", et c'est mystérieux. Ça moi c'est ça qui me plaît dans la musique et tous les albums que j'aime bien, je ne me suis pas vraiment renseigné sur la vie des mecs et tout ça je préfère la rêver, la fantasmer, me dire à tiens c'est bien il a fait ça... Et donc voilà tu vois, vu que c'est ça que j'aime, je vais pas m'épancher, faire un blogue, ou je vais dire "ah j'ai pensé ça, la vie..." (Paco, Pacovolume, pluriactif, rock alternatif, Paris)

Par surcroît, on peut remarquer que ce type de posture critique, vis-à-vis du caractère ostentatoire que la communication 
Compétences communicationnelles et pratiques numériques des « musiciens connectés »

médiatisée sur Internet peut engendrer, est assez courant chez les musiciens interrogés, même s'il ne se manifeste pas toujours sous la forme d'un jugement explicite et réflexif concernant les pratiques communicationnelles. Plus souvent, on a pu repérer cette critique sous la forme d'allusions ironiques ou sarcastiques, concernant des célébrités et leurs usages des réseaux socionumériques. Comme le montre cet échange avec les membres du groupe de rock anglais Kill Cassidy:

Martino - I guess Twitter is used more by sort of celebrities who, people are into know what they are onto every day, like Stephen Fry or so... people who got millions of followers in Britain.

Daniel - He can post that Stephen Fry is in the shower.

Martino - And then I hope he electrocutes himself. [Rires]

Daniel - And a million people "like" that". (Daniel et Martino, Kill Cassidy, pluriactifs, Rock alternatif, Bristol)

Corollaire de la logique d'action de promotion socialisante, la question de la pertinence des contenus cristallise ainsi une forme de double contrainte entre la nécessité de communiquer et la crainte corollaire de sa non-productivité ou, pire encore, de sa contre-productivité. Elle tente de reconstruire des frontières entre publications "pertinentes » (perçues comme " intéressantes », créatrices de liens ou d'implication de la part des fans) et publications «malvenues » ou « contingentes " (considérées comme du spam). Ici se confrontent également, à notre sens, deux univers de reconnaissance : la popularité (recherche d'un engagement avec un public) et la réputation (crainte de la réaction des pairs, notamment du fait de rendre visible la recherche d'interaction - et de reconnaissance -, dévoilant chez eux un caractère "superficiel » fortement dévalorisé par la critique artiste (Boltanski et Chiapello, 1999)).

Comme nous l'avons vu, pour certains musiciens, ces frontières sont très marquées et limitent de fait tout intérêt d'utiliser les réseaux socionumériques en dehors d'un usage purement promotionnel (et donc normatif), ce qui restreint

\footnotetext{
${ }^{8}$ Traduction libre : « Martino - Je suppose que Twitter est plus utilisé par les célébrités qui, dans l'activité de dire ce qu'ils font tous les jours, comme Stephen Fry ou alors... les gens qui ont des millions de followers en Grande-Bretagne./Daniel - II peut publier que Stephen Fry est dans la douche./Martino - Et alors j'espère qu'il s'électrocutera. [Rires]/Daniel - Et un million de personne "aime" ça. »
} 


\section{Stéphane COSTANTINI}

fortement l'utilisation d'un dispositif tel que Facebook pour ceux dont l'activité musicale est peu intense. Pour d'autres, ces frontières, perçues comme plus poreuses, semblent au contraire constituer une occasion pour étendre, affiner, voire affirmer, la production de leurs univers artistiques, en multipliant les initiatives et les collaborations artistiques ou médiatiques. Dans ce contexte, c'est davantage chez les musiciens portés par la volonté de saisir ces occasions que se mettent en place ce que l'on peut envisager comme de véritables "stratégies d'occupation du fil d'actualité » de Facebook, témoignant d'une tentative d'adaptation à la segmentation de l'attention sur le dispositif. En d'autres termes, et comme l'affirmait Beuscart (2008) à propos de MySpace, les musiciens « apprennent les règles qui gouvernent les interactions sur la plateforme ", règles parmi lesquelles la «tyrannie de la présence et de l'activité continue » (p. 25) prend une importance prépondérante pour les musiciens sur Facebook. Toutefois, les résistances à l'égard d'une marchandisation accrue des relations avec leurs auditeurs demeurent fortes, en partie par réaction au système du vedettariat - encourageant peu ou prou la mise en scène de l'image de l'artiste, ce qui les pousse a contrario à l'entretien d'un certain « culte de l'obscurité »-, mais également en raison d'une adhésion, héritée de la période romantique, à une vision traditionnelle de l'auteur séparé de son public.

Ce qui est en jeu dans la reconstruction de ces frontières, c'est la posture même du musicien en tant que " professionnel » - a contrario de celle de l' " internaute moyen " (ou de l'amateur), qui aurait une plus grande latitude quant à la pertinence des contenus qu'il publie. En ce sens, cette posture archétypale du professionnalisme tend à produire de nouveaux vecteurs de distinction sociale, tant sur le plan des usages que sur celui des discours qui les légitiment. Les tensions relevées dans les discours et la création de "limites ", de séparations, semblent prendre la forme d'une série de négociations réflexives dont la pratique amène l'objectivation et qui contribuent à l'acception progressive - bien que parfois distanciée - des dispositifs et des normes communicationnelles qui s'y instaurent.

Dans ces conditions, on peut affirmer que les dispositifs du Web collaboratif tendent à faire objectiver aux musiciens le sentiment qu'ils se disputent les mêmes ressources, dans la mesure où leurs auditeurs potentiels disposent d'une quantité 
Compétences communicationnelles et pratiques numériques des « musiciens connectés »

limitée de temps, de revenus disponibles ou allouables aux loisirs en général et à l'offre musicale en particulier. Cette objectivation peut apparaître comme un repoussoir pour les uns, comme un moteur dans la prise d'initiatives pour les autres, mais il semble en définitive qu'elle aboutit surtout à l'exacerbation des disparités liées aux compétences communicationnelles.

\section{Conclusion}

L'adoption progressive des dispositifs de distribution et de promotion en ligne par un ensemble grandissant d'acteurs des industries de la musique a activement concouru à la montée en puissance d'un phénomène d'injonction à la visibilité. La formation et le déploiement des compétences communicationnelles des musiciens concourent ainsi à ce que l'on peut désigner comme un " management de soi », incluant la gestion des activités de diffusion et de promotion musicale, mais aussi la gestion de leur visibilité et de leur image. Ce dernier point, dont on a vu l'aspect problématique, reflète plus largement des enjeux d'ordre socioanthropologiques concernant l'image de soi et son adaptation aux phénomènes de médiatisation sur Internet. Ces enjeux traversent et articulent à la fois les logiques d'intégration de l'artistique dans la communication, de socialisation de la promotion et, peut-être plus profondément, les modalités d'expression de leur subjectivité sur Internet. Chez les musiciens observés, le management de soi tend à couvrir à la fois l'apprentissage des articulations entre l'artistique et le communicationnel ainsi que la gestion publique/privée de leur personne - et le moins que l'on puisse dire est que ces articulations tendent à se complexifier avec l'appropriation des TIC.

Notamment, les injonctions à la recherche active et à l'entretien d'une "communauté de fans », supposant une posture du musicien en tant que gestionnaire de son image, entretenant des relations de connivence avec ses auditeurs et distillant des informations jugées personnelles (à l'instar du contenu de la presse people), sont souvent appréhendées avec une certaine circonspection. Elles manifestent en particulier les tensions entourant l'extension de marchandisation des contenus culturels se basant sur la gestion de leur image et sur les relations avec les auditeurs. Emblématique est, à cet égard, la 


\section{Stéphane COSTANTINI}

logique d'action d'" esthétisation de la contrainte communicationnelle ", envisagée comme une forme de " compromis » permettant une adéquation entre impératif communicationnel et travail artistique et qui paraît d'ores et déjà ancrée dans les pratiques. En ce sens, la déconstruction de la notion de visibilité nous paraît décisive :

- d'une part parce que cette notion, à l'acceptation floue, permet aux GAFAM de la constituer en produit d'appel, par le biais des nombreuses applications mettant en contact différents types d'audiences et de contenus, tout en monnayant les traces produites par leurs usages ;

- d'autre part, car, comme on a pu le voir, ces traces produites font appels à des univers de reconnaissance parfois en concurrence et dont les agents sociaux sont fortement différenciés aux yeux des musiciens, de même que leurs logiques d'actions reflètent un certain pragmatisme, tenant compte d'un développement plus large des trajectoires.

En outre, la professionnalisation des musiciens par la déspécialisation de leurs activités permet la mise en place de conditions favorables à la reproductibilité des pôles de production, notamment parce qu'il élargit les possibilités de gestion commerciale en interne de l'activité artistique. Cette reproductibilité se trouve tout à fait profitable aux acteurs des industries de la musique, mais également à ceux des industries du Web, car ces deux catégories d'acteurs ont de fait de moins en moins la tâche de contribuer financièrement à celle-ci, tout en conservant l'éventualité d'une cooptation pour les premiers et le maintien d'une relation profitable pour les seconds.

Enfin, on a pu voir que l'acquisition de nouvelles compétences communicationnelles tend à devenir un nouveau vecteur de distinction interne entre les musiciens. Or les compétences techniques, " commerciales » dans le cadre du webmarketing et des relations presse, évoluent très rapidement et l'offre de formations professionnelles à ces tâches et à destination des musiciens reste lacunaire. Cela pourrait donc, dans le même temps, renforcer le risque d'une "professionnalisation » à plusieurs vitesses, amplifiant les inégalités internes et l'incertitude quant aux chances de carrières stables. En d'autres termes, on assisterait à la fois au renforcement du brouillage 
Compétences communicationnelles et pratiques numériques des « musiciens connectés »

entre les "amateurs" et les "professionnels » et à l'accentuation de leur mise en concurrence sur Internet et (en particulier sur) les réseaux socionumériques dans le cadre d'une économie de l'attention où la demande n'est pas totalement illimitée.

Ces compétences deviennent un enjeu important puisque la mobilisation de la réputation des musiciens par les professionnels de la musique s'appuie de façon croissante sur la visibilité médiatisée des musiciens et se voit justifiée par le truchement des outils de calcul que les dispositifs médiatiques numériques mettent en place. Les artéfacts et les métriques relationnelles que ces dispositifs mettent en œuvre semblent alors en passe d'être naturalisés comme des indices d'une valeur symbolique, "virtuelle » mais dont les effets sont bien réels, qui n'attendrait plus que d'être actualisée par la vente des différents biens et services proposés.

On peut envisager, au terme de cette analyse, que c'est la relation entre les musiciens et leurs auditeurs, telle qu'elle se trouve médiatisée (et dans les conditions prévues) par les dispositifs numériques, qui tend à devenir un objet de la marchandisation - non sans résistances ni ambiguïtés du point de vue du secteur musical en général et des musiciens en particulier.

\section{Références}

Allard, L. (2009). Britney Remix: singularité, expressivité, remixabilité à l'heure des industries créatives. Vers un troisième âge de la culture?. Poli, (1). Repéré à https://polirevue.files.wordpress.com/2013/06/poli bonus lau rence-allard.pdf

Agamben, G. (2007.) Qu'est-ce qu'un dispositif?. Paris, France : Payot \& Rivages.

Belleflamme, P. (2003). Le piratage des biens d'information. Regards économiques, 12(17), 1-12.

Benghozi, P.-J. (2011). L'économie de la culture à l'heure d'internet : le deuxième choc. Esprit, juillet, 111-126.

Benghozi, P.-J. et Paris, T. (2001). L'industrie de la musique à l'âge internet. Gestion 2000, (2), 41-60. 


\section{Stéphane COSTANTINI}

Berger, V. (2011), Musique et stratégies numériques. Marketing, promotion, monétisation et mobilité. Paris, France : IRMA.

Bernoux, P., Amblard, H., Herreros, G. et Livian, Y. F. (2005). Les nouvelles approches sociologiques des organisations. Paris, France : Éditions du Seuil.

Beuscart, J.-S. (2007). Les transformations de l'intermédiation musicale : la construction de l'offre commerciale de musique en ligne en France. Réseaux, (141-142), 143-176.

Beuscart, J.-S. (2008). Sociabilité en ligne, notoriété virtuelle et carrière artistique. Réseaux, (152), 139-168.

Beuscart J.-S. et Couronné T. (2009). La distribution de la notoriété artistique en ligne. Une analyse quantitative de MySpace (enquête). Terrains \& travaux, (15), 147-170.

Boltanski, L. et Chiapello, E. (1999). Le nouvel esprit du capitalisme. Paris, France : Gallimard.

Bouquillion, P. et Matthews, J. T. (2010). Le Web collaboratif: mutations des industries de la culture et de la communication. Grenoble, France : Presses universitaires de Grenoble.

Bouquillion, P., Miège, B. et Mœglin, P. (2013). L'industrialisation des biens symboliques : les industries créatives en regard des industries culturelles. Grenoble, France: Presses universitaires de Grenoble.

Bullich, V. (2015). La propriété artistique comme régulation de la trivialité. Communication \& langages, (185), 67-88.

Citton, Y. (2012). Traiter les données: entre l'économie de l'attention et le mycélium de la signification. Multitudes, (49), 143-149.

Citton, Y. (2013). Le marketing entre économie de l'attention et exploitation culturelle. Dans P. Bourgne (dir.), Le marketing : poison ou remède ? Les effets du marketing dans une société en crise (p. 179-199). Paris, France : Éditions EMS.

Costantini, S. (2012). Création de la rareté : de la notion de vivier à l'ère du numérique. Dans J. T. Matthews et L. Perticoz (dir.), L'industrie musicale à l'aube du 21e Siècle - Approches critiques (p. 101-132). Paris, France : l'Harmattan.

Costantini, S. (2014). Les industries de la musique au prisme des acteurs de l'intermédiation numérique. Une analyse des 
Compétences communicationnelles et pratiques numériques des « musiciens connectés »

logiques socio-économiques et des pratiques communicationnelles des musiciens (Thèse de doctorat). Université Paris 13.

Coulangeon, P. (2004). L'expérience de la précarité dans les professions artistiques. Le cas des musiciens interprètes. Sociologie de l'art, 5(3), 77-110.

Coulangeon, P. (2008). Les transformations du marché du travail musical. Dans P. François (dir.), La musique : une industrie, des pratiques (p. 47-63). Paris, France: La Documentation française.

Dupuy-Salle, M. et Schmitt, L. (2019). Ulule, du courtage à la structuration des pratiques de création dans l'édition, la musique et l'audiovisuel, tic\&société, 13(1-2), 97-132.

Guibert, G. et Sagot-Duvauroux, D. (2013), Musiques actuelles : ça part en live. Mutations économiques d'une filière culturelle. Paris, France : Irma/DEPS.

Habermas, J. (1987). L'agir communicationnel. Tome I. Paris, France : Fayard.

Hesmondhalgh, D. (2007). The Cultural Industries. Londres, Royaume-Uni : Sage Publications.

Huet, A., Ion, J., Lefebvre, A., Miège, B. et Peron, R. (1978). Capitalisme et industries culturelles. Grenoble, France: Presses universitaires de Grenoble.

Jouvenet, M. (2006). Rap, techno, électro: le musicien entre travail artistique et critique sociale. Paris, France : Éditions $\mathrm{MSH}$.

Matthews, J. T. et Perticoz, L. (2012). L'industrie musicale à l'aube $d u \quad X X I^{e}$ siècle: approches critiques. Paris, France: L'Harmattan.

Matthews, J. (2015). Passé, présent et potentiel des plateformes collaboratives. Réflexions sur la production culturelle et les dispositifs d'intermédiation numérique. Les Enjeux de l'information et de la communication, (16), 57-71. doi: 10.3917/enic.018.0057

Menger, P.-M. (2009). Le travail créateur: s'accomplir dans l'incertain. Paris, France : Gallimard/Édition du Seuil. 


\section{Stéphane COSTANTINI}

Miège, B. (1989). The Capitalization of Cultural Production. New York, NY/Bagnolet, France : International General.

Negus, K. (1999). Music Genres and Corporate Cultures. Londres, Royaume-Uni : Routledge.

Nixon, B. (2013). Communication as capital and audience labor exploitation in the digital era (Thèse de doctorat). University of Colorado.

Shklovski, I. et Boyd, D. (2006). Music as Cultural Glue: Supporting Bands and Fans on MySpace. Repéré à http://www.danah.org/papers/BandsAndFans.pdf

Tschmuck, P. (2017). The US recorded music market in a longterm perspective, 1990-2016. Music Business Research. Repéré à https://musicbusinessresearch.wordpress.com/ 2017/05/05/3340/

Voirol, O. (2005). Les luttes pour la visibilité. Réseaux, (129-130), 89-121. 